\title{
BIÓPSIA PER-ORAL DO INTESTINO DELGADO EM 150 CASOS DE DIFERENTES ENTEROPARASITOSES *
}

\author{
Nortọ de Figueiredo **. Júlio Rubens **, Heleno Tinoco de Carvalho ** e I. Rodrigues da Silva ***
}

Os autores fazem uma apresentação sumária de alguns tipos de instrumentos utilizados na realização da biópsia duodeno-jejunal através intubação oral, ao mesmo tempo que comentam a importância do método no estudo da patologia intestinal.

Em seguida apresentam os resultados de sua experiência empregando 6 diferentes tipos de sondas e mostram os resultados histopatológicos observados em 150 casos de diversas parasitoses intestinais, submetidos a estudo. Entre os resultados chamam a atençäo para a presença freqüente da Giardia lamblia, detectada em 16 dos 50 (32\%) casos desta parasitose, contra 3 dos 27 (11,1\%) de es_ quistossomose e apenas 2 dos $108(1,8 \%)$ de estrongiloidiase.

Relatam também o achado de numerosas leishmânias no córion da mucosa jejunal de um paciente parasitado pela Giardia lamblia, que era portador de Calazar.

Apreciam, ainda, o significado de outras alterações, consideradas inespecificas, sôbre as quais aguardam estudos mais completos, para chegar a concíusões mais válidas. Entre estas, merece destaque o achado de atrofia das vilosidades intestinais em 27 pacientes infectados pelo Sirongyloides stercoralis sendo que em 5 dêles havia parasitismo exclusivo pelo referido nematóide.

\section{INTRODUÇÃO}

O uso de instrumentos para a realização de biópsia duodeno-jejunal, através de intubação oral, representa um grande progresso visando ao conhecimento da patologia do delgado, pois permite a obtenção de fragmentos da mucosa dêsse segmento intestinal, com um minimo de desconfôrto ou de risco para os pacientes.

Apesar da possibilidade de alterações traumáticas, seja pela ação de corte, seja pela sucção que é exigida pelo método, os detalhes histológicos mais delicados podem ser apreciados, quando se procede à fixação imediata dos fragmentos obtidos.

O estudo das alterações patológicas iniciais determinadas pelos parasitos que têm seu "habitat" no intestino delgado constitui grande indicação do método, que põe ao alcance do pesquisador achados que até então só eram possíveis através da autópsia ou da biópsia cirúrgica.

A chamada "síndrome pós biópsia" e as pequenas hemorragias, em sua maioria de significado desprezível, são as principais complicações do método. A perfuração e/ou as grandes hemorragias são de ocorrência bastante rara.

O processo de introđução de certas sondas pode às vêzes ser demorado; sua retirada pode ser penosa para o paciente, como acontece com a de CROSBY \& KUGLER, podendo mesmo ocorrer retenção da cápsula ao nível do piloro, o que em determinadas circunstâncias ocasiona sua separação do resto do instrumento. Em contraposição, há outros tipos de sondas, de manejo mais simplificado e sem os inconvenientes acima descritos.

O mecanismo que aciona o dispositivo de corte varia segundo o tipo de instrumento. Nas sondas de ROYER \& COLS., de SHINER, de BRANDBORG \& COLS., a lâmina é movimentada por um fio de aço, manejável do exterior, princípio que já fôra adotado na sonda para biópsia gástrica de WOOD. Outras modalidades empregam a

- Trabalho da Cadeira de Clínica de Doenças Tropicais e Infectuosas da Faculdade de Medicina da U.F.R.J., realizado em parte com o auxilio do "Grant AI-09188-0" do National Institutes of Health. Trabalho distinguido com o prêmio Gerhard Domagk da Sociedade Brasileira de Medicina Tropical, 1967

** Pical, 1967

*** Professor Catedrático 
QUADRO I - CARACTERISTICAS DOS INSTRUMENTOS DE BIÓPSIA INTESTINAL

\begin{tabular}{|c|c|c|c|c|c|c|}
\hline CARACTERISTICAS & $\begin{array}{l}\text { 1. ROYER \& COLS. } \\
\text { 2. SHINER }\end{array}$ & $\begin{array}{l}\text { 1. CROSBY \& } \\
\text { KUGLER } \\
\text { 2. CHELI \& COLS. }\end{array}$ & $\begin{array}{l}\text { 1. HENNING \& COLS. } \\
\text { 2. ROSS \& MOORE } \\
\text { 3. FLICK \& COLS. } \\
\text { 4. LEHMAN } \\
\text { 5. BAKER \& HUGHES }\end{array}$ & $\begin{array}{l}\text { 1. REICHARD \& } \\
\text { WESTERSTAHL } \\
\text { 2. BRANDBORG } \\
\text { \& COLS. }\end{array}$ & 1. CAREY & $\begin{array}{l}\text { 1. CHOUDHURY } \\
\& \text { COLS. }\end{array}$ \\
\hline $\begin{array}{l}\text { TAMANHO DA CÁP. } \\
\text { SULA }\end{array}$ & $\begin{array}{l}\text { 1. } 6 \times(30) \\
\text { 2. }(12 \times 20)\end{array}+$ & $\begin{array}{l}\text { 1. } 9.5 \times 18 \\
\text { 2. } 10 \times 20.5\end{array}$ & $\begin{array}{llll}\text { 1. } & 13 & \times 47 \\
2.9 & \times 28 \\
3 . & 8 & \times 24 & \\
4.11 & \times 34 & \\
5.8 & \times 12 \times 26\end{array}$ & $\begin{array}{llll}\text { 1. } & 13 & \times 24 \\
\text { 2. } & 4.6 & \times 12 & 12\end{array}$ & 1. $7 \times 20$ & 1. $9 \times 26$ \\
\hline $\begin{array}{l}\text { DISPOSITIVO DE } \\
\text { PROGRESSAO }\end{array}$ & $\begin{array}{l}\text { 1. FIO PONDE- } \\
\text { RADO } \\
\text { 2. BALAO }\end{array}$ & $\begin{array}{ll}1.0 \\
2.0\end{array}$ & $\begin{array}{l}\text { 1. BALÃO } \\
\text { 2. " } \\
\text { 3. SACO HG } \\
\text { 4. } 0 \\
\text { 5. } 0\end{array}$ & 1. $\overline{\mathrm{SACO} H G}$ & $\begin{array}{l}\text { 1. SACO } \\
\text { HG }\end{array}$ & 1. 0 \\
\hline $\begin{array}{l}\text { MOBILIZADOR DA } \\
\text { LAMMINA }\end{array}$ & 1. FIO DE AÇO & 1. MOLA & $\begin{array}{l}\text { 1. HIDRAULICO } \\
2 . \\
3 . \\
4 . \\
5 .\end{array}$ & 1. FIO DE AÇO & 1. SUCCAAO & 1. TRAÇÃo \\
\hline $\begin{array}{l}\text { NGMERO DE FRAG- } \\
\text { MENTOS RETIRA- } \\
\text { DOS POR OPERA- } \\
\text { CAO }\end{array}$ & $\begin{array}{l}\text { 1. MÚLTIPLO } \\
\text { 2. }\end{array}$ & $\begin{array}{l}\text { 1. UM } \\
\text { 2. }\end{array}$ & $\begin{array}{lc}\text { 1. } & \text { MULTIPLO } \\
2 . & " \\
3 . & " \\
4 . & " \\
5 . & "\end{array}$ & $\begin{array}{l}\text { 1. MÚLTIPLO } \\
\text { 2. }\end{array}$ & $\begin{array}{l}\text { 1. MÚLTI- } \\
\text { PLO }\end{array}$ & 1. MÚLTIPLO \\
\hline $\begin{array}{l}\text { INDICE DE SUCES- } \\
\text { SO }\end{array}$ & $\begin{array}{ll}\text { 1. } & 13 / 18 \\
\text { 2. } & 45 / 69\end{array}$ & $\begin{array}{l}\text { 1. } 90 \% \\
\text { 2. } 28 / 28\end{array}$ & $\begin{array}{ll}\text { 1. } & 80 \% \\
\text { 2. } & 18 / 20 \\
\text { 3. } & 80 \% \\
\text { 4. } & 11 / 12 \\
\text { 5. } & 34 / 36\end{array}$ & 1. $7 / 7$ & 1. $6 / ?$ & 1. $9 / 18$ \\
\hline
\end{tabular}

+ Os números entre parênteses são estimativos. (Adaptado de Crosby, 1963). 
pressão hidráulica ou mola, para mobilizar o mecanismo de corte (Quadro I - modificado de CROSBY - 1963).

$\therefore$ A penetração da mucosa no orifício da cápsula, onde funciona o dispositivo de corte, dá-se por sucção e o contrôle fluoroscópico se impõe como indispensável ao reconhecimento do local onde se vai operar, na maioria dos instrumentos.

A progressão da cápsula ao longo do intestino quase sempre é facilitada pela anexação de balōes cheios de ar ou mercúrio à sua extremidade distal.

O número de fragmentos retirados numa operação constitui outra característica importante do instrumento. No caso da sonda de CROSBY \& KUGLER, obtem-se um fragmento apenas, ao passo que, com os outros, pode-se conseguir número maior dêles.

As sondas que utilizam a corrente hidráulica permitem a obtenção dos fragmentos retirados imediatamente depois de seu corte, o que não se consegue com os outros tipos, que exigem a exteriorização do aparelho e abertura da cápsula, onde ficam retidos os referidos fragmentos. Entretanto, deve-se ressaltar que as sondas dêsse tipo são de introdução difícil, montagem e funclonamento complexos e muito mais sujeitas a falhas.

MATERIAL, METODOS E RESULTADOS DA TECNICA DE BIÓPSIA NA NOSSA EXPERIENCIA

No quadro I são encontradas as caracteristicas de algumas sondas e, no II, os resultados gerais obtidos com o emprêgo pessoal de 6 (seis) tipos diferentes de instrumentos. Os melhores resultados, quanto ao indice de sucesso, foram alcançados com as sondas de BRANDBORG \& COLS. $(\mathbf{8 0 , 8 \% )}$ e CROSBY \& KUGLER $(80 \%)$. evidente que $o$ resultado de nossas tentativas, até certo ponto discordante dos encontrados nas publicações de outros autores, deve-se, pelo menos em parte, à experiência do técnico que executa a biópsia. Com efeito, a sonda de CROSBY \& KUGLER, por exemplo, obteve apenas $65 \%$ de resultados positivos, com o técnico que iniciou seu emprêgo entre nós; ao passo que, em outras mãos, quando testado maior número de vêzzes, atingiu $80 \%$ de sucesso. Entretan. to, foi decidido o abandono do emprêgo dessa sonda, não sòmente devido a seu elevado preço, mas também porque fornece apenas um fragmento em cada operação e, quando de sua retirada, pode ficar retida ao nivel do piloro, retardando por trinta minutos, e às vêzes mais, a fixação do fragmento obtido.

Sôbre os resultados fornecidos pelà sonda de SHINER pode-se adiantar não serem compensadores, sobretudo por se tratar de instrumento grosseiro e volumoso, o que torna seu manejo bastante penoso, tanto para o operador como para o paciente.

Teòricamente, os instrumentos que utilizam a corrente hidráulica deveriam ser os mais recomendáveis; no entanto, nossa experiência, pelo menos com o modêlo de BAKER \& HUGHES, foi decepcionante.

Quanto à sonda de CAREY, por razões que desconhecemos, foi tão freqüentemente seguida de fracassos, que decidimos abandonar o seu uso.

Da mesma forma procedemos em relação ao instrumento de CHOUDHURY \& COLS., que não ofereceu um bom rendimento; além disso os fragmentos retirados apresentaram, por vêzes, alterações traumáticas decorrentes da tração exercida.

Por outro lado, a estratégia empregada com os diversos tipos de sonda tem variado. Assim, de início procedíamos sempre à anestesia do faringe, prática de há muito abandonada, por oferecer algum risco e nenhum proveito compensador. Também os antiespasmódicos foram ensaiados sem grandes vantagens, sendo que atualmente recomenda-se ao candidato apenas o jejum.

Com as sondas de SHINER, BAKER \& HUGHES, BRANDBORG \& COLS., a transposição do pilono se consegue, em geral, através de manobras transparietais sob contrôle fluoroscópico. No caso do aparetho de BRANDBORG \& COLS. conseguese, freqüentemente, a passagem espontânea pelo piloro, com o auxílio de pequeno balăo de mercúrio, adaptado à extremidade distal da cápsula. O instrumento de CROSBY \& KUGLER dispensa os cuidados acima descritos. A grande diferença de diâmetro entre sua cápsula e o tubo de polietileno que a comunica com o exterior, permite ao operador identificar a transposição do piloro pelo aumento de resistência 
à traçāo e seu progresso é garantido pelo peristaltismo intestinal.

Obtidos os fragmentos, procedem-se às recomendaçōes de SHINER \& DONIACH (1960), a fim de evitar cortes oblíquos, que possam dar uma falsa impressão de atrofia das vilosidades. Fazem-se em seguida os cortes histológicos em série, que são corados pela técnica da hematoxilina eosina, hematoxilina férrica, ou similares, consoante situaçóes especiais.

O intuito principal desta comunicação, entretanto, é apresentar os resultados histopatológicos do material obtido em 150 pacientes portadores de diferentes parasitoses, cujo habitat de eleição ou ocasional é $o$ intestino delgado.

Os enteroparasitos estão assim distribuídos:

Strongyloides stercoralis .. 108 casos

..Giardia lamblia ........ 50 "

Ancylostomidae ......... 45 "

Ascaris lumbricoides ..... 43 "

Schistosoma mansoni ..... 27 "

Essses parasitos foram encontrados quase sempre associados entre si e também a outros que habitam preferencialmente $o$ intestino grosso, como o Trichuris trichiura, - Enterobius vermicularis, a Entamoeba histolytica, etc.

Em alguns casos, detectamos apenas um único parasito do delgado, como se segue:

\begin{tabular}{|c|c|c|}
\hline Strongyloides stercoralis & 33 & casos \\
\hline Ancylostomidae & 12 & " \\
\hline Giardia lamblia & 8 & $"$ \\
\hline Ascaris lumbricoides & 4 & $"$ \\
\hline
\end{tabular}

Adotou-se como critério diagnóstico, em média, três coproscopias, utilizando as técnicas de HOFFMANN \& COLS., M.I.F.C., e BAERMANN. A biópsia retal foi também executada, a fim de evidenciar ou excluir a possibilidade de parasitismo pelo Schistosoma mansoni, nos casos com exames de fezes negativos para êsse trematóide.

Dos pacientes examinados, a maioria apresentava um estado geral regular ou bom, sendo alguns dêles assintomáticos $\mathbf{e}$ a maior parte com manifestaçōes clínicas discretas, como náuseas, dores abdominais leves e diárreia que se alternava ou não com a constipação intestinal. Poucos apresentavam anemia pronunciada ou evidência de outras carências graves.

Todos os pacientes apresentavam idade acima de 12 anos, distribuindo-se entre os diferentes sexos e raças, sem diferenças significativas.

Muitos dêsses pacientes submeteram-se à repetição da biópsia jejunal após tratamento especifico dos parasitos encontrados; entretanto, esta comunicação nāo tem o propósito de analisar comparativamente os dados dêsses exames, o que será objeto de um estudo completo, com avaliação das alterações clínicas e diferentes exames de laboratório, que incluem alguns testes de má absorção e exame radiológico do intestino delgado.

\section{RESULTADOS HISTOLÓGICOS E COMENTARIOS}

O exame histológico dos fragmentos de biópsia sempre permitiu reconhecer os di-

QUADRO II - NOSSA EXPERIENCIA COM 6 DIFERENTES TIPOS DE SONDA

\begin{tabular}{|c|c|c|c|c|c|c|}
\hline INSTRUMENTOS & SHINER & $\begin{array}{c}\text { CROSBY \& } \\
\text { KLUGER }\end{array}$ & $\begin{array}{l}\text { BRANDBORG } \\
\text { \& COLS. }\end{array}$ & $\begin{array}{l}\text { BAKER \& } \\
\text { HUGHES }\end{array}$ & CAREY & $\begin{array}{l}\text { CHOUDHURY } \\
\& \text { COLS. }\end{array}$ \\
\hline No dE TENTATIVAS $\ldots \ldots \ldots$ & 56 & $3180^{*}$ & 207 & 18 & 18 & 18 \\
\hline$+\ldots$ & - & $-\mathrm{e}-$ & - & - & - & - \\
\hline N: DE SUCESSOS & . $\quad 29$ & $20 \quad 64$ & 183 & 4 & 1 & 9 \\
\hline$\% \quad \ldots \ldots \ldots \ldots \ldots \ldots \ldots$ & . 51 & 65 e 80 & 80.8 & 22 & $: 5,5$ & 50 \\
\hline $\begin{array}{l}\text { No DE FRAGMENTOS POR OPE- } \\
\text { RAÇAO } \ldots \ldots \ldots \ldots \ldots \ldots \ldots\end{array}$ & $1-4$ & 1 & $1-4$ & $1-2$ & 1 & $1-2$ \\
\hline
\end{tabular}

- Voriações em função do téenico operador 
ferentes planos da mucosa intestinal e algumas vêzes até da submucosa. Todavia, como se sabe, a diferenciação entre as mucosas duodenal e jejunal nem sempre é fácil, pois, como foi assinalado por SHINER (1956-1957), aspectos estruturais peculiares ao jejuno podem corresponder aos últimos segmentos duodenais.

Como tem sido descrito em outras comunicações, o resultado mais constante dos estudos sôbre o assunto refere-se à infiltração monuclear do córion, acompanhada às vêzes de elementos eosinófilos e neutrófilos. Raramente encontramos proliferação conjuntiva do córion, algumas vêzes associada a pequenas hemorragias da submucasa, fato a que nāo atribuímos grande significado, tendo em vista que podem ocorrer em virtude do traumatismo determinado pela sucção, pelo corte ou pela tração do fragmento. E possivel que futuros estudos comparativos, analisando as diversas variáveis, permitam chegar a conclusōes sôbre o significado dessas alterações.

As células colunares do revestimento viloso não apresentavam modificaçōes, exceto determinado grau de distorsão; os núcleos estavam sempre preservados e nāo havia solução de continuidade, salvo quando surpreendidas estruturas estranhas, como um ôvo de Strongyloides stercoralis ou um granuloma subjacente. Em publicação recente RODRIGUES DA CIVA \& COLS. (1964), em estudo sôbre individuos parasitados pela Giardia lamblia, notaram certa exaltação da atividade muco-secretora, caracterizada por aumento numérico e volumétrico das células caliciformes.

Nesta comunicação a análise do material será dirigida a alguns aspectos especiais, pois julgamos atualmente que alterações como edema, congestão, infiltrado linfomonocitário, incremento da atividade mucosecretora e aumento das figuras de mitose devem ser analisadas segundo uma reavaliação completa baseada numa casuistica maior, que inclua pacientes sem parasitoses intestinais, para que se possa chegar a conclusões de maior valia.

No quadro III, são apresentados dados que nos parecem mais significativos e que se referem a:

\footnotetext{
A - Presença de parasitos

B - Atrofia das vilosidades

C - Infiltrado eosinofilico.
}

Os resultados que se referem à presença de parasitos revelam aspectos de interêsse:

a) Acentuada freqüência de Giardia lamblia nos fragmentos examinados - 16 entre 50 casos, ou seja $32 \%$; (Fig. 1) .

b) Rara incidência de Strongyloides stercoralis seja sob a forma de ôvo ou larva -2 entre 108 casos, ou seja 1,8\%; (Fig. 2).

c) Presença, aliás pouco freqüente, mas até certo ponto curiosa, de ovos de Schistosoma mansoni, em localização não considerada muito provável - 3 entre 27 casos, ou seja 11,1\%; (Fig, 3).

d) Em um dos casos com parasitismo pela Giardia lamblia, no qual se estabeleceu também o diagnóstico de Calazar em fase não avançada, pelo achado das Leishmania donovani na medula óssea e no fígado, foram detectados numerosos elementos em forma de leishmania no córion da mucosa obtida pela biópsia per-oral. (Fig. 4).

Mesmo analisando com detalhe os dois resultados não específicos já mencionados, isto é, a atrofia da vilosidade e a infiltração eosinofílica, ainda não podemos correlacioná-los com os fatôres etiológicos em jôgo.

Com relação à atrofia das vilosidades (Fig. 5), caracterizada pelo seu encurtar mento e alargamento, algumas vêzes assumindo o aspecto de clava, ou mesmo fusāo de diversos elementos nas suas camadas mais internas ou ainda adelgaçamento da zona contendo as glândulas de LIEBERKUNH (SHINER \& DONIACH, 1960), podemos dizer que foi observada em grau moderado em 5 casos de estrongiloitose, sendo que em 1 dêles havia fibrose da submucosa. Como êsses achados podem corresponder a quadros iniciais de má absorção, estamos aguardando uma reavaliaçāo do assunto, comparando os resultados antes e depois da terapêtica erradicante da parasitose, para um pronunciamento mais seguro a respeito do fato.

A propósito, convém lembrar que SHEEHY \& COLS. (1962) encontraram 
QUADRO III - ASPECTOS HISTOLOGICOS MAIS SIGNIFICATIVOS

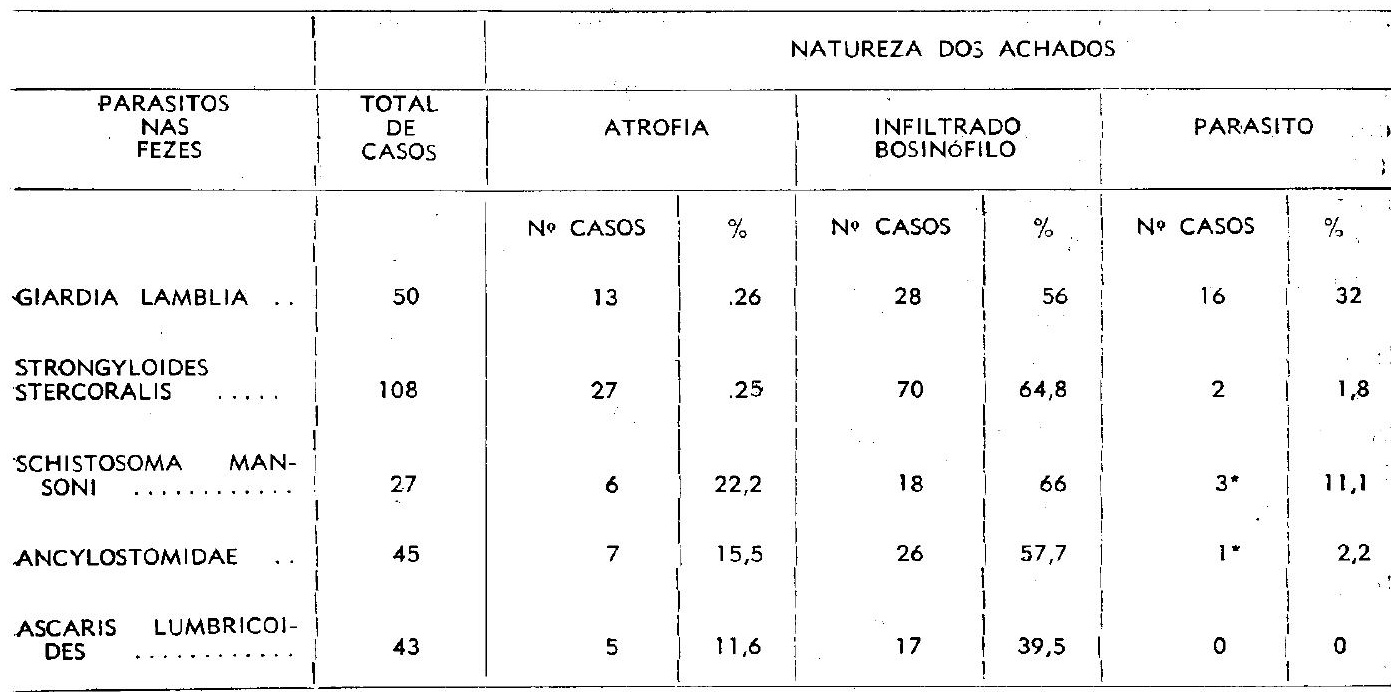

* Acolado ao fragmento de mucosa intestinal foi encontrade um exemplar macho de Necator americanus.

atrofia da mucosa intestinal em grande percentagem dos pacientes com ancilostomose, tendo sido observada a reversão das anormalidades, depois da erradicação da referida parasitose. Pelo menos em 7 casos de ancilostomose, do nosso material, havia atrofia parcial da mucosa do delgado, mas em todos êles registraram-se outras associaçōes parasitárias. O mesmo foi observaco em 5 casos de ascaridíase.
No que tange aos infiltrados eosinofílicos, foram observados com freqüência variável, mas de forma aparentemente mais significativa nos casos de estrongiloidiase. Convém assinalar sua presença em 15 dos 33 casos em que esta parasitose era exclusiva e também em 3 de 8 casos ùnicamente parasitados pela Giardia lamblia.

Voltando ao problema do aumento das figuras de mitose, alguns dos nossos resul-

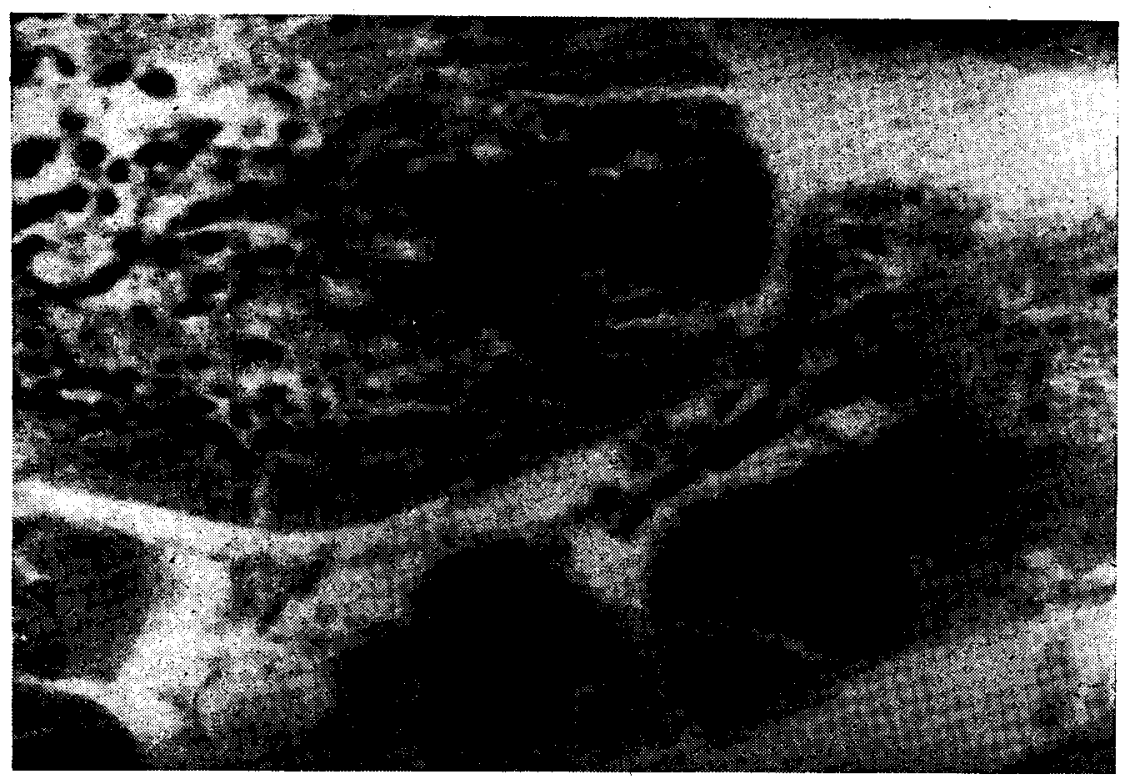

Fig. 1 - Trofozoitos de Giardia lamblia no mueo que recobre umo vilosidade (H.E., 400x). 


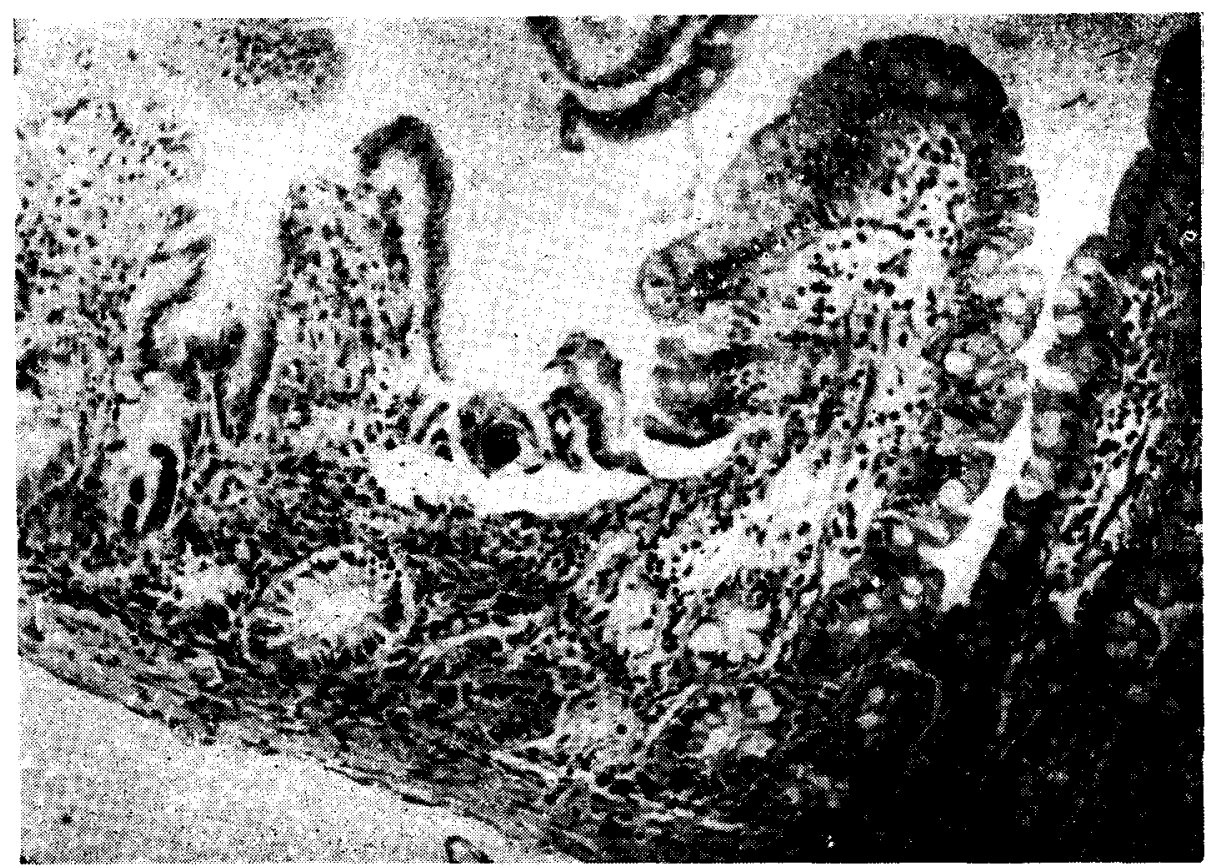

Fig. 2 - Strongyloides stercoralis. Óvo e larvo no epitélio glandular (H.E., 250x)

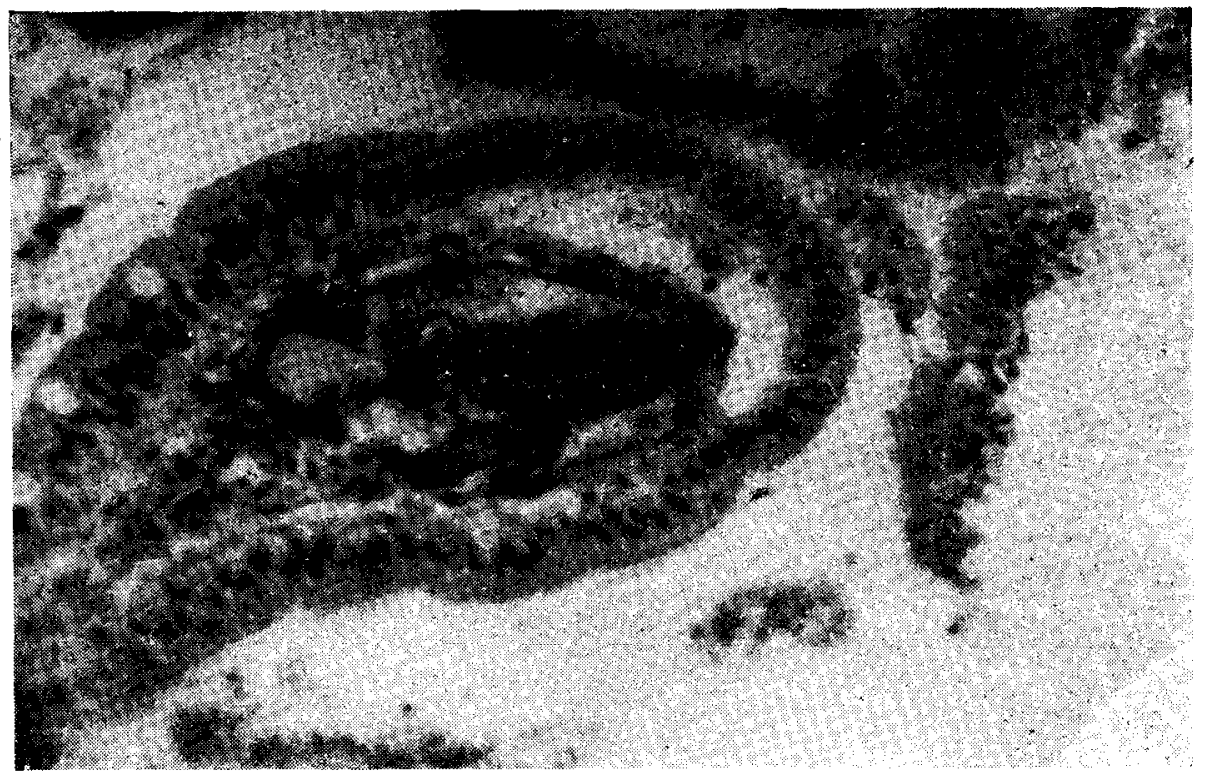

Fig. 3 - óvo de Schistosoma monsoni no corion da vilosidade (H.E., 400x: 


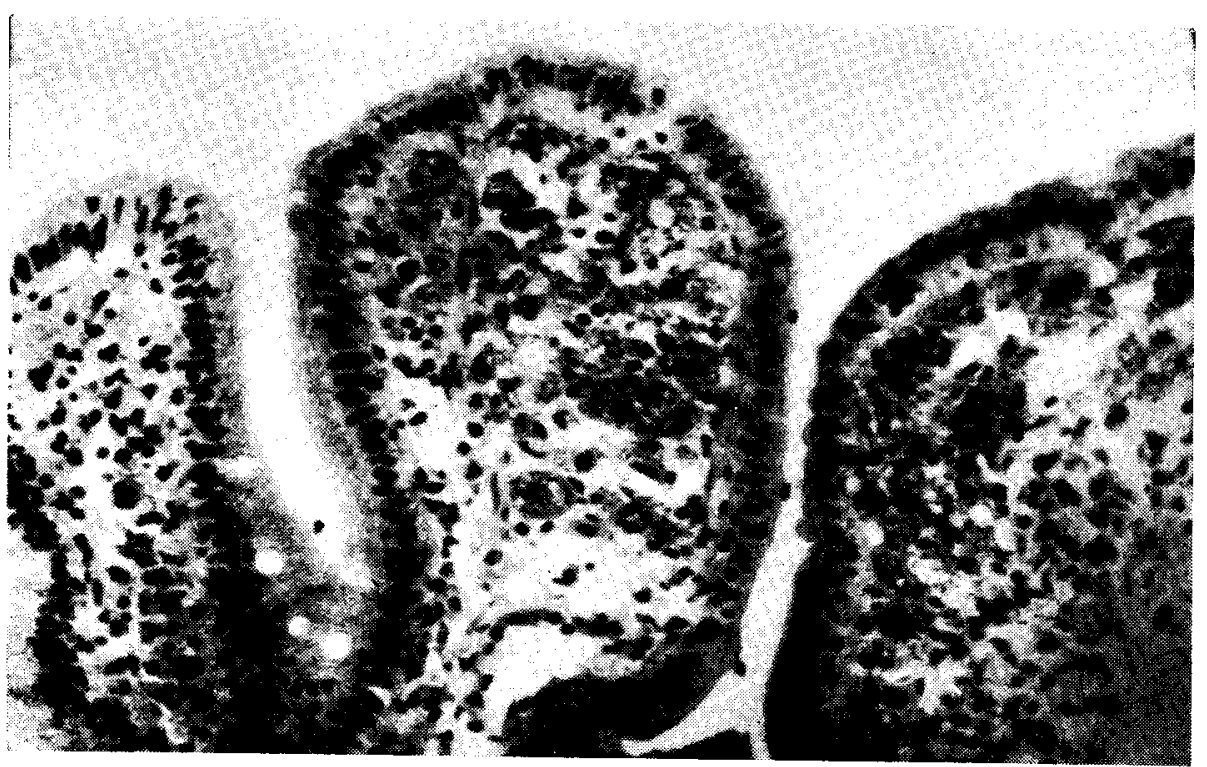

Fig. 4 - Leishmanias no interior de histiocitos do corion (H.E., 400 x)

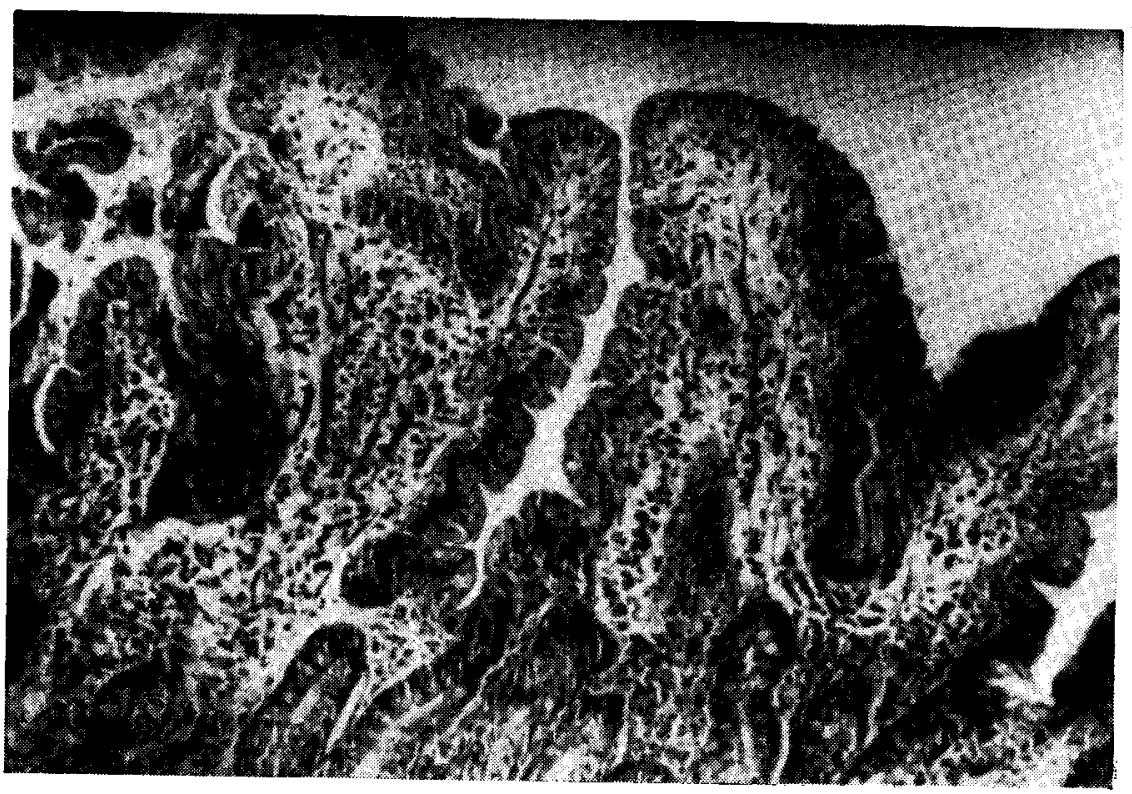

Fig. 5 - Atrofia parcial das vilosidades (H.E., 250x) 


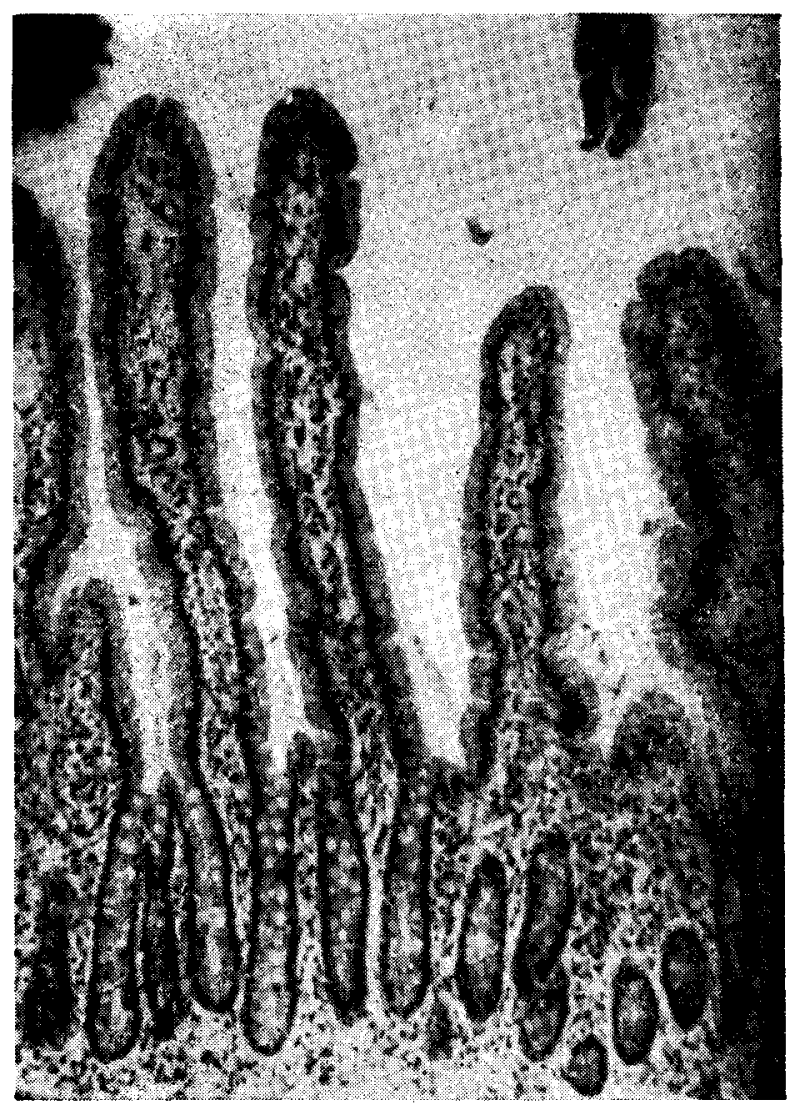

Vilosidades jejunais: padrão normal

tados, e mais freqüentemente dos casos de giardíase e estrongiloidíase, sugerem que o fenômeno possa ser relacionado à intensificação da atividade secretora ou à ativação do processo de renovação celular do epitélio intestinal. Parece ainda provável que na estrongiloidíase, e possivelmente na giardíase, haja também uma duodeno-jejunite catarral, que deve representar o estádio inicial da reação do órgão ao ataque do parasito.

Na giardíase, essa alteração seria a única a se processar, enquanto na estrongiloidíase a evolução do quadro atinge as formas de enterite edematosa e ulcerativa (DE PAOLA \& COLS., 1962).

De acôrdo com os resultados de FLOREY (1962) a secreção do muco pelo epitélio intestinal representa um mecanismo de de- fesa contra as agressões, pôsto que evita o contato direto do parasito com a mucosa. No caso da Giardia lamblia e outros parasitos da luz intestinal, êste mecanismo é preponderante, conforme resultados recentemente publicados (RODRIGUES DA SILVA \& COLS., 1964). Na estrongiloidiase e esquistossomose, como os elementos parasitários situam-se principalmente na intimidade dos tecidos, a intensificação do mecanismo mucosecretor se torna, talvez, menos necessária.

Com relação aos resultados comparativos das biópsias, antes e depois da terapêtica antiparasitária, podemos no momento adiantar que houve desaparecimento da Giardia lamblia do fragmento de todos os casos nos quais o parasito fol encontrado antes da terapêutica específica. 


\author{
S U M M A R Y
}

The authors describe some types of capsules used in their experiences and point out the value of small intestine biopsy to the study of intestinal pa:hology. Six different types of capsules were tried.

Hystopathological findings in 150 patients infec'ed with different entersparasites are described. Giardia lamblia was found in the mucosa in 16 out of 50 cases of parasitism by the protozoa; in $11.1 \%$ of the cases of schistosomiasis and $1,8 \%$ of those of strongyloidiasis parasites were seen in the mucosa. A case is described of a patient with Giardia lamblia and kala-azar; leishmanias were seen in the corion.

Some other hysthological changes, considered as non specific, need more detailed studies in order to give valuable conclusions; atrophy of villi, e.g., was found in 27 cases infected by Strongyloides stercoralis, 5 of them exclusively infected by this helminth.

\title{
BIBLIOGRAFIA
}

1. BAKER, S. J. \& HUGHES, A. - Multiple-retrieving small-intestinal biopsy tube. Lancet, 2: 686, 1960.

2. BRANDBORG, L. L., RUBIN, G. E. \& QUINTON, W. E. - A multipurpose instrument for suction biopsy of the esophagus, stomach, small bowel, and colon. Gastroenterology, 37: 1, 1959.

3. CAREY, J.B., JR. - A simplified gastrointestinal biopsy capsule. Gastroenterology, 46: 550, 1964.

4. CROSBY, W.H. \& KUGLER, H.W. Intraluminal biopsy of the small intestine. The intestinal biopsy capsule. Am. J. Digest. Dis., 2: 236, 1957.

5. CROSBY, W. H. - Small intestinal studies. Methods for obtaining intraluminal contents and intestinal mucosa. A review. Am. J. Digest. Dis., 8: 2,1963 .

6. DE PAOLA, D., DIAS, L.B. \& RODRIGUES DA SILVA, J. - Enteritis due to Strongyloides stercoralis. Report of 5 Fatal Cases. Am. J. Digest. Dis., 12: $1086,1962$.

7. FLOREY, H.W. - The secretion and function of intestinal mucus. Gastroenterology, 43: 326, 1962.

\section{RODRIGUES DA SILVA J., COUTI-}

NHO, S.G., DIAS, L.B. \& DE FIGUEIREDO, N. - Histopathologic Findings in Giardiasis: A Biopsy Study. Am. J. Digest. Dis., 9: 5, 1964.

9. ROY-CHOUDHURY, D.C. COOKE, W. T., SMITS, B.J. \& BANWELL, J.E. Simple capsule for multiple intestinal biopsy specimens. Lancet, II: 185, 1964.

10. ROYER, M., CROXATTO, O., BIEMPICA, L. \& BALCAMAR MORRISON, A.J. - Biopsia duodenal por aspiración bajo control radioscópico. Prensa Méd. Argent., 42: 2515, 1955.

11. SHEEHY, T.W., MERONEY, W.H., COX, R.S.J. \& SOLER, J.E. - Hookworm disease and malabsorption. Gastroenterology, 42: 148, 1962.

12. SHINER, M. - Duodenal biopsy. Lancet, 1: $17,1 / 956$.

13. SHINER, M. - Duodenal and jejunal biopsies. Gastroenterology, 33: 64, 1957.

14. SHINER, M. \& DONIACH, I. - Histopathologic studies in steatorrhea. Gastroenterology, 38: 419, 1960.

15. WOOD, I.J., DOIG, R.K., MOTTERAM, R. \& HUGHES, A. - Gastric Biopsy. Report on fifty-five biopsias using a new flexible gastric biopsy tube. Lancet, 1: 18, 1949 . 